\title{
EL GRAN LUHMANN
}

THE GREAT LUHMANN

RESUMEN: Esto texto ha sido producido en homenaje a los vinte años de la publicación del libro sociedad de la sociedad que ocurrió en 2007.

Palabras clave: Teoria de sistemas. Niklas Luhmann.
ABSTRACT: This text has been produced in tribute to the twenty years of the publication of the Society Society book that occurred in 2007.

Keywords: Systems theory. Niklas Luhmann.

\footnotetext{
${ }^{1}$ Profesor de tiempo completo Departamento de Ciencias Sociales y Políticas, Universidad Iberoamericana Ciudad de México. Miembro del Sistema Nacional de Investigadores (SNI), Nivel III. Posdoctor con beca otorgada por la fundación Heinrich Hertz de la República Federal Alemana, en la Universidad de Bielefeld. Traductor de obras de Niklas Luhmann al espanol.
} 


\section{INTRODUCIÓN}

En un último estudio sobre la circulación del pensamiento europeo, Leandro Rodríguez Medina postula que en la recepción del pensamiento de Luhmann en Hispanoamérica podrían distinguirse tres círculos: a) un primer círculo de receptores mucho más interesado en el contexto general de la teoría) un segundo más preocupado por la aplicación empírica de la teoría y c) un último círculo de utilización hibridada de la teoría (MEDINA, 2014). Con alto grado de arbitrio podría elevarse este esquema a destino de todo gran pensamiento: el momento especulativo, el empírico, el hibridado. Podría incluso postularse que estos tres momentos son necesarios para el 'sistema'. Quisiera concentrarme en el carácter especulativo de la teoría de Luhmann. Así como en la sociología es consigna estructural la necesidad de aplicación empírica, el pensamiento de Luhmann adquiere una especial relevancia a la luz de su carácter teórico. El mismo Luhmann lo anuncia en el inicio del libro sistemas sociales:

Como ciencia empírica, la sociología no puede prescindir de la aspiración a comprobar sus afirmaciones mediante los datos obtenidos de la realidad, independientemente de qué tan viejos o nuevos sean los conductos por donde se vacía lo ya ganado. Justamente en razón de este principio, no puede fundamentar el campo específico de su objeto ni la unidad propia de su disciplina científica. La resignación ha ido tan lejos que ya ni siquiera se hace el intento (LUHMANN, 1998, p. 7).

No sólo allí, Luhmann finalizó sus famosas lecciones sobre la introducción a la teoría de sistemas diciendo: "La intención de estas lecciones era la de enfrentar precisamente a la tradición humanista y de proponerse la pregunta de si hay teorías con capacidad de responsabilizarse ante un rompimiento de tales dimensiones. Espero que estas lecciones hayan logrado hacer ver que eso es posible". Y luego Habermas en distintos momentos expresó: "Luhmann, inspirado en Maturana y otros, ha extendido y flexibilizado hasta tal punto los conceptos básicos de la teoría de sistemas, que ha logrado convertir ésta en base de un paradigma filosófico capaz de competir con los demás" (HABERMANS, 1990, p. 32). Luhmann, pues, puesto en referencia a la teoría. En expresión del mismo Luhmann: "podría incluso renunciarse a la razón si se supiera cómo podría salvarse el interés por la 
reflexividad teórica. Porque en este siglo hay muy pocos ejemplos de interés decidido por la teoría".

Mi tesis es muy cercana a la sostenida por Daniel Chernilo: Lo que caracteriza la gran historia de la sociología de Luhmann es una pretensión universalista del conocimiento. "La pretensión universalista puede describirse por un triple movimiento: 1) definir conceptualmente en qué consiste los social de las relaciones modernas 2) formular metodológicamente cuáles son los mejores procedimientos para estudiar lo social de manera fiable 3) justificar normativamente la idea de que la sociedad moderna es una y sólo una" (CHERNILO, 2011, p. 242). Sin embargo, lo específico de mi argumento es llamar la atención sobre la relevancia de la configuración teórica del pensamiento de Luhmann.

\section{EL ARGUMENTO DE LA COMPLEJIDAD}

En Luhmann la teoría tiene que ver con la dimensión hipercompleja del mundo: el investigador siempre se encuentra con que hay más cosas que las que puede aprehender, más huellas que las que puede rastrear, más posibilidades que las que puede 0 selección. Lo que lo hace distinto a otro tipo de actores es la manera en que organiza dicha selectividad. Si esto se entiende en su justa dimensión, entonces se vuelve comprensible la conceptuación de Luhmann sobre la técnica: la técnica podría considerarse como simplificación que funciona. Se trata de un proceso efectivo de aislamiento, de desentenderse de la híper-complejidad del mundo. La técnica es altamente efectiva pero a costa de no involucrar las consecuencias no deseadas. Esto sugiere la conjetura de que el incremento del saber sobre la naturaleza lleva tan sólo el incremento del no-saber sobre las consecuencias de las intervenciones técnicas. La teoría en cambio se sitúa en otro contexto: en el problema de manejar la sobrecarga de complejidad y las posibilidades de selección. La teoría se planta precisamente en el punto de descarga de la complejidad y entonces Luhmann es capaz de afirmar por ejemplo: "la diferenciación funcional no puede llevar a cabo (contra todo lo que afirma la auto-descripción de los sistemas) la total inclusión postulada. Los sistemas funcionales (cuando operan racionalmente) excluyen a las personas de manera tan fuerte que esto les trae consecuencias para el acceso a otros sistemas... Si este diagnóstico es más o menos acertado, la sociedad no puede esperar de 
la sociología ni consejo ni ayuda. Pero podría tener sentido al ofrecer teorías que hacen más justicia (más justicia que el depósito tradicional crítico-optimista de nuestra disciplina) a los hechos - hechos que, sobre todo, la misma sociedad produce" (LUHMANN, 1996, 219-230).

Por la complejidad, la teoría no es sólo cuestión de 'neutralidad valórica' para darse después vuelo disparando juicios al objeto y no a la teoría misma. La complejidad puede hacer añicos los juicios críticos desde el momento en que la sociedad no opera bajo un continuum de racionalidad. Por la complejidad, la sociología proyectiva (cuya capacidad de juicio lo obtiene a partir de un juicio inconsiderado de lo posible) encuentra que los contextos causales entre causas y efectos son sólo apropiados para sistemas relativamente pequeños. Y lo mismo podría decirse de la investigación estadística: en los sistemas altamente complejos se encuentra con altos grados de dispersión azarosa en las grandes cantidades, es decir, con un alto grado de falta de estructura. Los grandes sistemas no se encuentran determinados por su estructura, sino que resuelven lo que para ellos es posible a través de su historia. No son, pues, máquinas triviales.

La complejidad extrema es justamente el núcleo de la sociedad moderna $-\mathrm{y}$ es esta complejidad la peculiaridad de su referencia al mundo. La propuesta de Luhmann es escoger el problema de la complejidad como último punto de referencia del análisis y considerar todos los sistemas como aprehensión y reducción de complejidad y, en esa perspectiva extremadamente abstracta, considerarlos como comparables e intercambiables. La unidad de dicha teoría de sistemas estaría en la unidad de los problemas de base que están presentes en todos los sistemas. Para la praxis de la teoría, los contornos de una teoría así podrían bosquejarse de la siguiente manera:

1) Muchas veces la manera clásica de interponer los problemas llega a callejones sin salida. Las grandes posibilidades de éxito no están en la dirección de buscar la solución de los problemas sino en el sentido contrario: buscar un mayor nivel de abstracción. Luhmann para ilustrar esto recurrió a un ejemplo espléndido: en la baja Edad Media ante el cambio inminente de la positivización (volverse mudable) del derecho, se trató de hacer que el derecho siguiera fiel a la tradición obligando a los señores a prestar juramento. Y justo el derecho pudo hacerse positivo, como hoy lo 
entendemos, renunciando a la base duradera que dichos juramentos podían conferirle.

2) La situación práctica de la teoría se vuelve viable para el teórico en la medida en que hace que su teoría se ocupe de la complejidad. Esto evidentemente no conduce con obviedad a un conocimiento 'práctico' en el sentido de rico en acciones. Pero construye un entendimiento tal de complejidad en la teoría, que la teoría puede aconsejar mejor e interpretar mejor al práctico. De lo que se trata es poner en juego un contexto más rico en alternativas y mucho más complejo.

\section{LA TEORÍA Y EL DIÁLOGO CON OTROS GRANDES PARADIGMAS MODERNOS}

\subsection{La fundamentación pragmático trascendental}

La teoría de Luhmann puede compararse con otros grandes paradigmas modernos de fundamentación metodológica de lo social. Con su teoría sobre la sociedad se pone a la par en la historia de la filosofía con la más radical aplicación del convencionalismo (los juegos lingüísticos). Los seres humanos no utilizan un sistema onto-semántico de un lenguaje ideal que suponga a priori la determinación de sentido de las proposiciones, sino que el uso que hacen los seres humanos de los signos se debe al sentido como médium de lo social. No sólo el significado de los signos depende de la regla de su aplicación, también el sentido de las reglas de aplicación depende en todo momento de las convenciones que se establezcan en la sociedad. Con su teoría de la sociedad, Luhmann no solo ha anulado todos los criterios de validez metafísico-objetivos, sino también las condiciones subjetivas de posibilidad de la objetividad en sentido kantiano. Precisamente porque no existe ninguna garantía metafísico-objetiva o subjetiva del sentido de los signos o de la validez de las reglas, la sociedad se vuelve el horizonte de todos los criterios de sentido y validez. Nosotros los seres humanos (como seres dotados de lenguaje) estamos condenados a concordar en los criterios de sentido y validez impuestos en el acto social. Aunque pudiera objetarse que el acuerdo lingüístico en cada juego posible está vinculado a priori a reglas que posibilitan las convenciones, estas meta-reglas de todas las reglas establecidas convencionalmente no pertenecen a determinados juegos lingüísticos o formas de vida, sino al sentido emergente de la ilimitada comunidad de comunicación: la sociedad. 
Si se presupone la separación sujeto/objeto (tradicional en la filosofía cartesianokantiana) no puede pensarse la sociedad sin contradicción. Deberíamos recordar que han negado también la relación sujeto/objeto tanto la filosofía del Espíritu de Hegel como las ciencias histórico-hermenéuticas. Prescindiendo de las diferencias significativas epistemológicas que existen entre estos enfoques, la sociedad es para ellos un sujetoobjeto con el que nos podemos identificar mediante comprensión. Incluso esto podría incluir a Marx, quien también parte de un concepto de sujeto/objeto. ¿Qué relación guarda, pues, la noción de sentido en Luhmann con la fundamentación dialécticohermenéutica tradicional de las ciencias del espíritu o sociales?

Según lo menciona Karl Otto Apel (1985, p. 237 e s.s.), Peter Winch, en the Idea of a social science and its relation to philosophy, es el primero en plantear la relación, p. ej., entre juego lingüístico y la problemática fundamental de las ciencias sociales. La pregunta que plantea es: cómo sabemos que un individuo cumple la regla que nosotros atribuimos desde afuera? Y él da la respuesta: podemos comprender cualquier serie de acciones con distintas fórmulas, con tal de que se escoja una fórmula suficientemente compleja: sólo puedo constatar que un ser humano sigue una regla con sentido si su comportamiento resulta comprensible a partir de un juego lingüístico como seguimiento de reglas controlables públicamente. Participar en un juego lingüístico común es el camino que se abre para la superación sujeto/objetos que sólo puede exigirse por completo a las ciencias sociales, y que no pudieron resolver en el siglo xix debido a la teoría sicologista de la comprensión qua empatía o rememoración. Evidentemente el postulado de la sociedad como horizonte es una solución diferente de aquella que resuelve el problema del acuerdo intercultural basándose únicamente en la semejanza entre condiciones vitales humanos semejanza que es fáctica y constatable mediante comparaciones empírico-antropológicas. En pocas palabras: la auténtica condición de posibilidad del acuerdo estaría constituida por la sociedad, que ciertamente tiene su base real y su punto de partida genético en los hechos fundamentales de la vida humana generalizada. Puesto que (con Wittgenstein) es impensable el lenguaje privado, los pensamientos y acciones solitarios de cada individuo están ya siempre relacionados con el juego del lenguaje en la comunidad ilimitada de comunicación. 
A la larga sólo puede alcanzarse esta meta de la filosofía y las ciencias sociales críticas, realizando prácticamente la comunidad ilimitada de comunicación de los sistemas sociales. La propuesta de Luhmann se distingue de la filosofía trascendental kantiana en la medida en que no considera la unidad de la conciencia del objeto y de la auto-conciencia como punto supremo. Si la argumentación en cuanto tal ha de tener sentido, esta unidad de la interpretación tiene que poder alcanzarse, por principio, en la comunidad ilimitada de los que se comunican. En esta medida, la propuesta se entiende como transformación, desde la crítica del sentido, de la filosofía trascendental, que parte del factum a priori de la argumentación como un punto de arranque cuasi-cartesiano insuperable. El orden emergente del sentido puede mostrar que la aceptación de las reglas de juego de una comunidad crítica de la comunicación es algo praxeológico, condición de posibilidad y validez de la comprobación de los hechos - propia de las ciencias empíricas.

\subsection{Ampliación de las disposiciones comunicativas en vez de Institución: vs. Gehlen}

En el contexto de la teoría de Luhmann existe una evidente referencia a la antropología cultural de Gehlen ${ }^{2}$. La antropología provocativa de Gehlen establece: estando dotado el ser humano de tanta plasticidad e inestabilidad de las pulsiones cómo es que llega a desarrollar una conducta instintiva y automática. Y su respuesta es: gracias a las instituciones. Gehlen transfiere los problemas más agudos de la ética a la situación humana originaria de la sujeción de los instintos. Esto lo toma de la teoría de Konrad Lorenz: en los animales existe una conducta análoga a la moral, es decir, instintos inhibitorios conservados por la especie -instintos que impiden atentar contra el semejante indefenso. Cuando falta esta capacidad inhibitoria se observan en la especie humana fenómenos como el canibalismo extendido entre los hombres primitivos y las guerras de extinción de las comunidades étnicas en la actualidad. Lo que está detrás del

\footnotetext{
${ }^{2}$ Luhmann titula uno de sus primeros libros: Los derechos fundamentales como Institución. "El último asistente de Gehlen atribuye a Luhmann motivos científico-políticos para distanciarse de la herencia gehliana. Luhmann no quiso, por razones de reputación, ser identificado con un representante de la 'escuela sociológica de Leipzig'. En efecto, Gehlen -después de haber encontrado su modus vivendi al lado de Adorno- en 1969, con una crítica moral y un diagnóstico del tiempo basado en la teoría de la decadencia, puso en su contra no sólo a Habermas sino también a su primer asistente Schelsky, el apoyador de Luhmann, haciendo una profesión de fe por las instituciones (Lepenies 2009)" (LUHMANN, 2010, p. 27).
} 
redescubrimiento antropológico de las Instituciones en Gehlen es la idea fundamental de que la orientación sensorial y los desencadenadores específicos externos deben sustituirse por Instituciones. Gehlen desconfía de las teorías de la subjetividad y da un viraje de la antropología a la filosofía de la historia.

Deducir formas del espíritu objetivo (como el cristianismo puritano o la ética confuciana) directamente de la subjetividad humana es para Gehlen precipitado. Las instituciones son una especie de astucia de la razón que logra de refilón en los seres humanos una elevación indirecta sobre sí mismos. De ello se sigue metodológicamente hablando que preguntarse por las formas del espíritu objetivo es algo que sólo puede hacerse desde un punto de vista sociológico-cultural, es decir, preguntando al mismo tiempo por las Instituciones. Entre la conciencia subjetiva y sus contenidos de sentido fáctico median las instituciones como idea directriz.

Gehlen llega a la generalización filosófica de que las instituciones son las formas supremas del orden y del destino que nos amparan y nos perfeccionan. Los seres humanos se entregan a ellas con un grado de libertad mayor que aquel que tuvieran actuando por sí mismos. "Y las instituciones como el matrimonio, la propiedad, la iglesia o el Estado libera a los hombres precisamente de su propia subjetividad inmediata, prestándoles una subjetividad más elevada que atraviesa los designios del mundo y de la historia, aunque también les protegen de sí mismos, dándoles opción, sin exigírselo, a un ajustamiento de la subjetividad supremo e incomparable" (APEL, 1985, p. 199-200).

En Luhmann, en cambio, no son las instituciones sino las disposiciones comunicativas funcionalmente diferenciadas, las que confieren fijación frente a la alta contingencia humana. Partiendo de esta premisa, el desarrollo social que se extiende hasta nuestros días puede entenderse como ampliación de las disposiciones comunicativas "pero no como una ampliación del ser humano en el sentido de Rousseau o Nietzche" (LUHMANN, 1993, p. 42). A partir de grandes logros culturales, las posibilidades de éxito de la comunicación se impulsaron hasta lo inverosímil, porque "ningún ser humano concreto puede generar por sí mismo individualidad, civilización, dinero o poder legítimo" (LUHMANN, 2010, p. 308). A ello contribuyeron dos principios: la diferenciación de una determinada capa superior, dotada de una fácil y atinada comunicación interna (p.ej., la 
nobleza) y la diferenciación de determinados centros de gravedad funcionales, que en un principio eran sobre todo de tipo religioso, político-militar y comercial. "La diferenciación de religión, política, economía y formación de la personalidad empezó a florecer apenas en la Edad media tardía, gracias a que la reflexión filosófica había alcanzado en la escolástica un grado de abstracción que facilitó la discusión crítica y el cambio social de las premisas. La consecuencia fue una ola de fanatismo religioso-reformador que, sin embargo, no se tradujo en instituciones estables, sino en una guerra religioso-confesional, que se fue apagando en los inicios del tiempo moderno" (LUHMANN, 2010, p. 80).

Sobre esta base la sociedad moderna adquiere su perfil particular, históricamente único. Se caracteriza por el primado de la diferenciación funcional, que tiene el efecto de una enorme ampliación de las comunicaciones altamente especializadas, así como de su efectividad: lenguajes extremadamente teóricos como las matemáticas, la multiplicación del empleo de la técnica en la comunicación por parte de los medios de masas, la institucionalización de medios de comunicación simbólicos, como el amor, el dinero, el poder, etc. (LUHMANN, 2010, p. 72). De esta forma, el potencial para la acción humana se puede organizar, especializar y coordinar de un modo como no había sido posible hasta ahora. La verdad comprobada científicamente, el dinero, el poder organizado por la política y acotado por el derecho, sirven de mecanismos para llegar a actuar, incluso en aquellos casos en los que el ser humano, desde una perspectiva antropológica, nunca lo hubiera logrado. Lo improbable se convierte en posible, se vuelve rutinariamente pronosticable: puede acumular sus propios efectos, acrecentarse a sí mismo y, con todo ello, tiene profundos efectos sobre el eco-sistema de la sociedad e incluso sobre el mismo ser humano.

\subsection{Dignidad de la sociología como 'sociología primera': con Husserl vs. Husserl}

El afán sempiterno de la filosofía (la aprehensión de esencias) parecía que se lograba por fin en la fenomenología de la manera más sencilla. La filosofía no había llegado a ser ciencia rigurosa porque siempre modeló su concepto de ciencia según el modelo de las ciencias particulares: la filosofía debe partir inversamente de la crítica a los métodos y a 
los fundamentos de aquellas para así volverse capaz de ofrecer un verdadero sistema y no visiones parciales.

A partir de allí, la tarea histórica que vio Husserl fue elevar la fenomenología a la dignidad de filosofía primera. Por mucho que los conceptos matemáticos sean psicológicos en su origen, las leyes que por ellos se expresan no son psicológicas, no dependen de la constitución psíquica del hombre. Las matemáticas presentan un contenido no empírico y no temporal. En todos estos casos se trata de una validez a-priori y absoluta que no puede provenir del pensamiento, sino de su objeto.

Todo el problema está en determinar si el objeto (la esencia) tiene validez en sí o solamente para una conciencia y de aquí viene el giro de Husserl hacia lo trascendental. A todo fenómeno psíquico corresponde, pues, por la vía de la reducción fenomenológica un fenómeno puro, que exhibe su esencia inmanente (singularmente tomada) como dato absoluto. La conciencia se convierte en Husserl en el único ente absoluto a partir del cual toda realidad alcanza su sentido. Por ente absoluto entiende Husserl ese ente que, en forma de una vida intencional y tenga lo que tenga presente en su conciencia, es a la vez conciencia de sí mismo. Y esta capacidad de autorreflexión es lo que caracteriza a la conciencia o subjetividad trascendental como realidad absoluta.

El mundo consiste en el redescubrimiento de la génesis intencional, por lo que este mismo mundo se fue constituyendo en el sentido de la conciencia. Es decir, la conciencia por su misma existencia presupone la existencia de la realidad - realidad por otra parte que no tiene sentido para el hombre sino a la luz de una conciencia que la descubre. No hay inmanencia sin trascendencia, no hay interioridad sin exterioridad, no hay yo sin mundo. E inversamente: no hay trascendencia sin inmanencia, no hay exterioridad sin interioridad, no hay mundo sin yo.

Luhmann responde:

Lo duro de esta despedida del sujeto trascendental puede reconocerse en cuanto se piensa si es posible que la conciencia pueda abandonarse como único médium de la construcción de formas y, a pesar de ello, conservar la estructura descubierta por Husserl, es decir, el entendimiento del nexo condicional entre capacidad de operación, separación y proceso simultáneo de heterorreferencia y autorreferencia, así como el de una temporalidad a partir de cada uno de los momentos operativos. Lo tengo por posible desde el momento en que uno se decide partir del sentido como médium general para la construcción de formas, 
con el fin de distinguir si los sistemas se construyen en razón de una intencionalidad de la conciencia o en razón de la comunicación. Para el caso de las prestaciones de la conciencia podrían repetirse los análisis de Husserl, aunque ya no se necesitaría caracterizarlos como trascendentales. Para el caso de la comunicación debe encontrarse una construcción paralela que documente que aquí funciona tan bien como en el caso de la conciencia. Es decir, sólo como operación actual momentánea -como establecimiento de límites, como procesamiento simultáneo de heterorreferencia y autorreferencia (por tanto como bi-estabilidad) y, yendo más allá, como recursos recursivos y anticipativos en un tiempo inactual aunque aprehensible dentro de un horizonte temporal de pasado y futuro y, sobre todo: inclusión de lo excluido como modo de procesar el sentido.

Pienso que un programa de teoría así, que distingue de manera radical entre sistemas psíquicos y sociales, es realizable - aunque aquí no sea el momento de mostrarlo. ${ }^{3}$ La pregunta sería: ¿cómo se vería el campo de la teoría si este programa fuera realizable?

Tendríamos un tipo de diseño teórico que no se articularía a partir de leyes de la naturaleza (tipo antiguo), ni de sus derivados estadísticos, ni del leitmotiv de la eficacia de los acoplamientos técnicos. La crítica de Husserl a la parcialidad de la idealización galileo-cartesiana y de su forma vinculante en las matemáticas se sostendría. Tampoco tendríamos una teoría dialéctica (independientemente de su valoración positiva o negativa) que concluyera en un fin alcanzable. Tampoco sería una tabla de cruzamientos de variables -en el sentido parsonianoderivada del concepto de acción. No sería ninguna lógica que trataría de responder desasiéndose de las paradojas, sino más bien una teoría que mantuviera abierta la paradojización y desparadojización de su distinción directriz, para el caso de que ya no fueran convincentes las formas que ella ofrece. Sería una teoría de los sistemas autorreferenciales, no-triviales, por tanto movedizos e incalculables, que tienen que delimitarse de su entorno para lograr su tiempo y sus valores-propios y así llegar a delimitar sus posibilidades. Una teoría que asumiría la tarea cibernética de controlar su propia indeterminación (LUHMANN, 1997).

\section{EL INSTRUMENTAL BÁSICO-TEÓRICO PARA ENTENDER LA ILIMITADA COMUNIDAD DE}

\section{COMUNICACIÓN}

\subsection{El medio emergente del sentido}

Respecto a otras tradiciones de pensamiento, el concepto de sentido goza en Luhmann -si pudiera decirse así- de un estatuto especial. En el plano del sentido no se trata ni de información ni de significados ni de cultura, sino de cómo se lleva a cabo, en absoluto, en el mundo, la constitución de sentido. Es el médium más general e insuperable que posibilita los sistemas psíquicos y sociales. Su perspectiva garantiza que el mundo se

\footnotetext{
${ }^{3}$ Véase para un itento así Niklas Luhmann, Sistemas Sociales: Lineamientos para una teoría general, Anthropos/Uia/Ceja, Barcelona, 1998. Véase también Heinz von Foerster, Für Niklas Luhmann: Wie rekursiv ist Kommunikation? Teoria Sociologica 1/2 (1993), pp. 61-85, con la afirmación decisiva de que la comunicación es recursividad.
} 
haga accesible y es la condición y el ámbito donde se procesa el sentido de manera temporal. Se trata, pues, de un orden emergente superior:

El sentido es la "sustancia" propia de este plano emergente de la evolución... Sería un desacierto tratar de encontrar un portador del sentido. El sentido se soporta a sí mismo en la medida en que -refiriéndose-a-sí-mismo- posibilita su propia reproducción... Es cierto que para la constitución del sentido son necesarias condiciones previas evolutivas altamente complejas, pero no existe ningún portador privilegiado, ningún sustrato óntico del sentido (LUHMANN, 2007, p. 114-115).

Luhmann sugiere (respecto a la reflexión sobre lo social) si no seríamos más afortunados suponiendo que lo social está orientado por un dinamismo comunicativo de sentido, frente al cual el ser humano se encuentra formando parte del entorno. Esta hipótesis se acomoda más a la posibilidad, siempre esbozada por la sociología, de que lo social es un orden emergente. Los seres humanos, al estar situados en el entorno frente a este dinamismo de sentido, no pueden ya sin más ser concebidos como una especie de tribunal superior que juzga el destino colectivo. Por lo contrario, los hombres se hacen dependientes de este dinamismo de sentido en el caso de que deseen establecer contactos sociales.

¿Qué significa que los seres humanos sean entorno de este dinamismo de sentido? Ser entorno, para Luhmann, es ser fuente inagotable de estimulación, de irritación, de perturbación, pero nunca, de manera causal directa, fuente de determinación. Entre la comunicación y la conciencia se da una relación asimétrica. Para Luhmann (a pesar de Marx) ni el orden social determina la conciencia ni la conciencia el orden social.

Entre las diversas conciencias no puede formarse en sentido estricto nada en común de suerte que la vivencia de ego pudiera llegar a coincidir punto por punto con la experiencia de alter. Lo que surge, en cambio -y eso es un sistema social- es un desarrollo del sentido que surge por auto-reproducción, y que para desarrollarse tiene necesidad de establecer límites (clausurarse), con respecto a la vivencia de cada conciencia individual. Los sistemas sociales reproducen el sentido bajo la forma de comunicación. Y la comunicación introduce una pluralidad de perspectivas sobre el objeto, de tal suerte que nunca es posible lograr una única perspectiva común. La comunicación es, pues, por razones estructurales, diversidad de perspectivas sobre el objeto. 
Para superar esta diversidad de base de lo social, la evolución ha echado mano del lenguaje. Sin embargo, el lenguaje no pone a disposición algo así como expresiones con significado idéntico, sino que sólo permite sustituir el sentido por signos. Lo cual hace surgir la ilusión de la unidad de perspectivas que se tendrían sobre el mundo. El lenguaje no ofrece suelo sólido alguno sobre el que ego pudiera reunirse en un consenso definitivo con alter.

Por eso, la comunicación (forma-de-sentido) es la estructura última de lo social y aquello que posibilita el que se den incluso perspectivas antónimas sobre el mundo. La divergencia es, así, la estructura más íntima de lo social y ha sido necesaria una inversión descomunal de energía para lograr, mediante evolución, puntos de coincidencia, acuerdos, consensos -los cuales siempre serán contingentes.

La sociedad es, por consiguiente, pura comunicación. Sería muy improbable, sobre todo con la carga de conocimiento moderno sobre la incomprensibilidad del otro, que los seres humanos pudieran hacerse dependientes entre sí, mediante acuerdos permanentes. Por esta razón las teorías contractuales de la sociedad han caído en desuso. En todo caso, si los individuos han de sacar alguna ventaja de la convivencia humana, se hacen dependientes más bien de un orden superior con cuyas condiciones pueden elegir los contactos recíprocos y, precisamente por esto, son mínimamente dependientes unos de otros. Para los seres humanos este sistema de orden superior es el sistema de comunicación llamado sociedad: "En otras palabras, debe existir en el plano del sistema emergente un modo propio de operar (aquí la comunicación) una propia autopoiesis, una posibilidad auto-garantizada de continuidad de las operaciones; de otra manera, la evolución de las posibilidades del vicarious learning nunca hubiera resultado exitosa" (LUHMANN, 2007, p. 148).

Así como los seres humanos viven en la dependencia estructural de las leyes físicas y aprenden a sacar consecuencias de esta dependencia, de la misma manera los hombres viven en la dependencia estructural de un cosmos de comunicación. Evidentemente que entre las estructuras cósmicas y las estructuras de comunicación media un abismo de flexibilidad. Mientras que la ley de la velocidad de la luz es insuperable, los sistemas sociales de comunicación están caracterizados por su enorme flexibilidad estructural: la 
flexibilidad de los programas políticos; la flexibilidad de los medios masivos de comunicación que cambian permanentemente; la flexibilidad del derecho positivo; la flexibilidad de los recursos monetarios. En una palabra: la flexibilidad de que disponen los sistemas sociales en términos de cultura y de memoria constituyen la verdadera ventaja de nuestra sociedad.

\title{
4.2 Emergencia
}

La afirmación de que el sentido está instalado en un plano emergente está puesta en la mismísima dirección de Popper (1975, p. 30-31): "Según las opiniones cosmológicas actuales, entre los sucesos emergentes más importantes se encuentran quizás los siguientes [...]: a) la cocción de los elementos más pesados b) el comienzo de la vida sobre la tierra c) la emergencia de la conciencia d) la emergencia del lenguaje humano y del cerebro humano". Popper (1975, p. 23) además explica lo que entraña esto que se designa como emergente:

\begin{abstract}
En contra de todas estas opiniones, sugiero que el universo, o su evolución, es creador y que la evolución de los animales sentientes con experiencias conscientes ha suministrado algo nuevo. Al principio dichas experiencias eran de tipo más rudimentario y, posteriormente, de un tipo superior. Finalmente surgió esa especie de conciencia del yo y ese tipo de creatividad que, según sugiero, encontramos en el hombre... A lo que me refiero con la palabra 'creativo' es a lo que se refiere Jacques Monod (1970-1975) cuando habla de la impredictibilidad de la emergencia de la vida sobre la tierra, de la impredictibilidad de las de las diversas especies y particularmente de nuestra propia especie humana [...] éramos impredecibles antes de que apareciésemos. La idea de evolución 'creadora o emergente es muy simple y un tanto vaga. Alude al hecho de que en transcurso de la evolución ocurren cosas y sucesos nuevos con propiedades inesperadas y realmente impredecibles. Estos sucesos o cosas nuevas contra toda idea reduccionista no pueden explicarse causalmente a partir de los niveles más bajos (POPPER, 1975, p. 17-18).
\end{abstract}

\subsection{La distinción}

En el plano emergente del sentido sólo se participa de dos maneras: introduciendo distinciones (operación de la comunicación) y percibiendo las distinciones (operación de la conciencia).

Con el concepto de sentido Luhmann toma posición en cuanto a que la vivencia y la acción se encuentran de alguna manera estructuradas: se remiten a otras posibilidades de 
vivencia o de acción. El sentido (así en Luhmann) supone un contexto de remisión de la actual vivencia y acción a otras posibilidades de vivencia o acción o a otras posibilidades de distinguir o de observar. Con el concepto de forma Luhmann toma posición sobre otra condición sin la cual no fuera posible la vivencia o la acción: éstas están siempre dirigidas hacia algo determinado. Pensamos en un determinado objeto, actuamos con una determinada intención hacia un fin expreso o hablamos sobre un tema específico. El presupuesto de que nosotros nos dirijamos a algo determinado es que podamos distinguirlo, a saber, distinguirlo de todos los otros posibles objetos o de otros objetos determinados. Entonces, para que podamos intentar algo determinado es necesario distinguir. Y se llega a la distinción a través de que nosotros intentemos algo determinado o, como Luhmann lo formula, mediante la designación de algo explícito o que marquemos un lado de la distinción. Esta es la quintaesencia del concepto de forma. Sólo podemos designar algo en la medida en que lo distingamos, y podemos distinguir algo cuando lo señalamos. Cada distinción remite a dos lados: uno que queda indicado, y otro que queda no-indicado. La unidad de la distinción indicación/distinción será llamada forma. Este pensamiento se lo debe Luhmann a la obra proto-matemática de Georg Spencer Brown. En su escrito sobre "las leyes de la forma" Spencer Brown se refiere a la pregunta de cómo en absoluto se forman las identidades de sentido. $Y$ Spencer Brown afirma: se forman mediante las distinciones e incluso estas distinciones anteceden a todos los cálculos (teoremas, (números, identidades) de los lógicos.

Si Husserl anduvo tras la idea de que más allá de cada vivencia intencional de los seres humanos se encontraban leyes elementales de la vivencia (subjetividad en la forma más pura) Spencer Brown cree haber dado con un mecanismo todavía más elemental, el cual antecede a toda determinación de la vivencia, a saber: la distinción. Piensa él que las formas matemáticas (que comienzan con la distinción) son expediente especial porque representan y se derivan de los modos internos del pensar: tienen tanta validez como el conocimiento mismo de la realidad externa: "La disciplina de las matemáticas es vista como camino de revelación de nuestro conocimiento interno de la estructura del mundo", y esto es además "aviso directo de que la forma matemática es estructura arquetípica" (BROWN, 1972, p. xvii; xx). Así el campo de objetos de la sociología tiene que ver con las 
formas de observar distinciones. La sociología en su aspecto esencial tiene que ver con observaciones de sentido que observan otras observaciones de sentido en sus formas actuales. Con esto se agota el objeto de la sociología. Su tarea consiste en preguntarse por la condiciones bajo la cuales estas observaciones se realizan y se estabilizan haciendo posible la construcción del mundo social ${ }^{4}$.

\section{LA REFLEXIÓN SOCIAL DE LUHMANN COMO ‘SOCIOLOGÍA PRIMERA’}

En la tradición de pensamiento occidental, Aristóteles impuso un estilo de pensar referido a la filosofía primera; en griego: $\pi \rho \omega \tau \eta$ ciencia que se ocupa de las realidades que se encuentran por encima de las realidades físicas. Por eso, posteriormente, se ha llamado metafísica a toda tentativa del pensamiento humano dirigida a trascender el mundo empírico para alcanzar la realidad meta-empírica. De manera similar habría que entender la reflexión de Luhmann como 'sociología primera': tratar de delimitar en su forma más básica aquel ámbito emergente del mundo que llamamos sociedad. Es evidente que aparte de la sociedad existen otros muchos ámbitos: el hombre, la naturaleza, los organismos vivos, el cosmos... Desde una perspectiva formal Luhmann pretende que la sociología (ciencia que se aboca al fenómeno social) indague: 1) Los principios primeros y supremos del orden social 2) analice la operación constitutiva de la socialidad; 3) estudie la comunicación -que es la sustancia de la socialidad y 4) investigue la sociedad, que es el fenómeno omnicomprensivo de todo lo que se designa como social.

La sociología, pues, de Luhmann se presenta en primer lugar como la búsqueda de los principios primeros y supremos del orden social (formas elementales).

Descubre que el principio fundamental (y primero de la sociedad) se encuentra en el hecho de que la sociedad es tan sólo forma. Forma -como se expresó en el apartado anterior - es simplemente la paradoja que resulta del empleo de una distinción. Es paradoja porque la distinción juega con dos movimientos simultáneos: 1) juega con la unidad al incluir algo; y 2) juega con la diferencia excluyendo algo.

\footnotetext{
${ }^{4}$ Véase esto (puesto aquí como resumen) más extensivamente en Rainer Schützeichel, Sinn als Grundbegriff bei Niklas Luhmann, Campus, Fankfurt/Main, 2003, cap. 2.
} 
Si quisiéramos una definición moderna del hombre siguiendo el estilo de Aristóteles, se podría condensar probablemente de esta manera: el hombre es el animal que emplea distinciones. El ser humano se alza por encima de todo lo demás del mundo, porque juega con distinciones. Y, para señalarlo de nuevo, distinguir es jugar con la unidad y simultáneamente con la diversidad. Así como en la mitología el rey Midas con tan sólo tocar las cosas las convertía en oro, así nosotros con sólo nombrar las cosas las partimos en un juego paradójico de unidad y diferencia. Al señalar las cosas, el juego que todos jugamos es un juego de inclusión y exclusión.

Es evidente que no todo lo que experimentamos lo traducimos en distinciones. Incluso podría afirmarse que la manera elemental en la que experimentamos el mundo transcurre sin distinción. Estamos en el mundo. Tenemos una identidad primaria. Vivimos. Experimentamos el mundo. El problema, sin embargo, irrumpe cuando todo eso lo queremos comunicar, por tanto cuando lo queremos hacer social. Entonces la experiencia originaria del nosotros y del mundo se tiene que traducir en distinciones.

Por tanto podemos ya concluir que el concepto más amplio y extenso de la sociedad -la fuerza centrípeta de todo lo social- es la operación por medio de la cual introducimos distinciones.

La sociedad es un cálculo de distinciones, un juego de distinciones. Un juego que separa, discrimina, discierne, unidades y crea por eso mismo diferencias. La sociedad es, pues, en su principio y fundamento un cálculo formal: una forma.

En el transcurso de la evolución este cálculo formal se traduce en la operación de la comunicación. Los seres humanos nos comunicamos recurriendo a una forma: a un código, a un lenguaje, a señas, a símbolos. La comunicación es en cierto sentido la forma -la naturaleza íntima - de la sociedad. La sociedad está compuesta tan sólo por formas de comunicación.

Dentro de estas formas no hay nada material, nada orgánico, nada del ser humano. La sociedad es tan sólo forma.

Las cinco características decisivas de la sociedad son:

1. La sociedad es un dinamismo de formas de comunicación. 
2. Este dinamismo existe como un orden de reproducción emergente separado del orden de reproducción de las condiciones físico-químico-orgánico-espirituales que lo posibilitan.

3. Mediante estas formas de comunicación, la sociedad -en el transcurso del tiempo- va adquiriendo contornos de más determinación.

4. La sociedad (la forma) es una unidad múltiple compuesta de partes - que a su vez obedecen a un mecanismo emergente. La sociedad no es, pues, un todo compuesto simplemente de partes, sino un todo compuesto de partes emergentes.

5. La sociedad es un dinamismo formal entre lo actual y lo posible. O para decirlo de una manera más fuerte en alusión a la teoría de la sustancia de Aristóteles: la sociedad no tiene realidad ontológica propia. No es más que una abstracción formal y que existe sólo como símbolo entre los seres humanos.

\section{口a}

\section{REFERENCIAS}

APEL, Karl Otto. La transformación de la filosofía I. Madrid: Taurus, 1985.

APEL, Karl Otto. La transformación de la filosofía II. Madrid: Taurus, 1985.

CHERNILO, Daniel. La pretensión universalista de la teoría social. Santiago: LOM, 2011.

FOERSTER, Heinz von. Für Niklas Luhmann: Wie rekursiv ist Kommunikation? Teoria Sociologica, v. 1/2, 1993, p. 61-85.

HABERMAS, Jürgen. Pensamiento postmetafísico. México: Taurus 1990.

LUHMANN, Niklas. Die neuzeitlichen Wissenschaften und die Phänomelogie. Wiener Vorlesungen: Picus, 1997.

LUHMANN, Niklas. Jenseits von Barbarie. In: Maxi Miller; Hans-Georg Soeffner (eds.). Modernität und Barbarei. Soziologische Zeitdiagnose am Ende des 20. Jahrhundert, Frankfurt: Suhrkamp 1996, p. 219-230.

LUHMANN, Niklas. La sociedad de la sociedad. México: Herder/UIA, 2007

LUHMANN, Niklas. Los derechos fundamentales como institución. México: UIA, 2010.

LUHMANN, Niklas. Politische Soziologie. Berlín: Suhrkamp, 2010.

LUHMANN, Niklas. Sistemas Sociales: Lineamientos para una teoría general, Anthropos/Uia/Ceja, Barcelona, 1998. 
LUHMANN, Niklas. Teoría política en el Estado de Bienestar. Madrid: 1993.

MEDINA, Leandro Rodríguez. The Circulation of European Knowledge (Niklas Luhmann in the Hispanic Americas), Palgrave Pivot, 2014.

SCHÜTZEICHEL, Rainer. Sinn als Grundbegriff bei Niklas Luhmann. Fankfurt/Main: Campus 2003.

SPENCER-BROWN, George. Laws of Form. Nueva York: The Julian Press, p. xvii y xx.

NAFARRATE, Javier Torres. El gran Luhmann. RBSD -

Revista Brasileira de Sociologia do Direito, v. 5, n. 2, p. 6-

24, mai./ago. 2018.

Recebido em: 18/02/2018

Autor convidado 